\title{
Response surface methodology-based hybrid robust design optimization for complex product under mixed uncertainties
}

\author{
WAN Liangqi, CHEN Hongzhuan, and OUYANG Linhan* \\ College of Economics and Management, Nanjing University of Aeronautics and Astronautics, Nanjing 211106, China
}

\begin{abstract}
Minimizing the impact of the mixed uncertainties (i.e., the aleatory uncertainty and the epistemic uncertainty) for a complex product of compliant mechanism (CPCM) quality improvement signifies a fascinating research topic to enhance the robustness. However, most of the existing works in the CPCM robust design optimization neglect the mixed uncertainties, which might result in an unstable design or even an infeasible design. To solve this issue, a response surface methodology-based hybrid robust design optimization (RSM-based HRDO) approach is proposed to improve the robustness of the quality characteristic for the CPCM via considering the mixed uncertainties in the robust design optimization. A bridge-type amplification mechanism is used to manifest the effectiveness of the proposed approach. The comparison results prove that the proposed approach can not only keep its superiority in the robustness, but also provide a robust scheme for optimizing the design parameters.
\end{abstract}

Keywords: response surface methodology (RSM), hybrid robust design optimization (HRDO), uncertainty, complex product of compliant mechanism (CPCM).

DOI: $10.21629 /$ JSEE.2019.02.10

\section{Introduction}

Owing to the compact size and the large amplification ratio of the compliant mechanism, it has been widely used in the micro-electro-mechanical-system (MEMS) for transforming an input displacement at one port to an output displacement at another port through elastic body deformation [1,2]. Compared with the traditional rigid-body mechanism, the compliant mechanism has numerous merits in the performance improvement and cost reduction, e.g., simplify manufacturing processes, decrease assembly

\footnotetext{
Manuscript received March 15, 2018.

*Corresponding author

This work was supported by the National Natural Science Foundation of China $(71702072 ; 71811540414 ; 71573115)$, the Natural Science Foundation for Jiangsu Institutions (BK20170810), and the Ministry of Education of Humanities and Social Science Planning Fund (18YJA630008).
}

time and reduce mechanical friction. Therefore, the compliant mechanism revolutionary changes the mechanical engineering and disciplines. Nowadays, existing studies for the complex product of compliant mechanism (CPCM) have a range of fruitful research achievements in the robust design optimization [3]. However, most of the existing studies in the compliant mechanism typically have a crucial drawback, which only consider the aleatory uncertainty in the robust design optimization. Li et al. [4] noted that neglecting the mixed uncertainties (i.e., the aleatory uncertainty and the epistemic uncertainty) in the robust design optimization could lead to an infeasible design. Therefore, there is a strongly increasing requirement to take mixed uncertainties into consideration for optimizing the CPCM. In addition, although the input-output functional relationship for the quality characteristic might be established based on physical mechanisms, the underlying mechanical model cannot be precisely obtained for the CPCM due to the difficulties in interpretation. Therefore, a response surface methodology (RSM) modeling technique is adopted to construct an accurate empirical model for the CPCM in the robust design optimization. Consequently, one of the most challenging issues is the robustness enhancement in CPCM, which is also one of research topics in the industrial engineering field. The purpose of this paper is to develop an RSM-based hybrid robust design optimization (HRDO) approach for the CPCM, which takes into account the mixed uncertainties and the accurate empirical model. The main contribution of this paper is that we propose an integrated HRDO approach for the CPCM by considering the mixed uncertainties.

The robustness improvement for the CPCM is invariably realized by using the robust topology optimization approach, which regards uncertain structure parameters as random design variables in the robust topology optimization. Wang et al. [5] explored a new robust topology approach for the compliant mechanism with the desired 
motion output by minimizing the expectation of Taguchi quantity loss function. Wittwer et al. [6] investigated an approach for the preliminary stage design model validation, the uncertainty analysis and the robust design optimization in the nonlinear compliant micro-mechanism to account for the critical process uncertainties including material stiffness, residual stress and edge bias. Lazarov et al. [7] provided a novel large-displacement compliant mechanism robust design optimization, in which the design variations are modelled with the help of random variables. Garcia-Lopez et al. [8] rendered an improved robust topology optimization approach, which deals with the robustness issue in the mechanical structure by using multi-objective evolutionary algorithms. Dao et al. [9] proposed a novel approach to the robust optimal parameter design for a compliant microgripper, which couples the hybridization of Taguchi with the differential evolution algorithm. According to the existing approaches, the CPCM robust design optimization under the mixed uncertainties is still in the primary stage. Most of these studies on the CPCM are restricted to the robust topology optimization approach, which only assumes the design variable as a random variable (i.e., the aleatory uncertainty). The existing approaches seem not to be good choices, since neglecting the mixed uncertainties might result in a vulnerable optimum structural design or an infeasible structural design. Furthermore, most of the studies employ the pseudo-rigidbody model to construct an input-output functional relationship for the CPCM. It is worth mentioning that an accurate approximation cannot be obtained via the pseudorigid-body model when the CPCM is very complicated.

In recent years, there have been several approaches dealing with the uncertainties in the design optimization, which mainly classify two approaches (i.e., the probabilistic approach and the interval approach). The probabilistic approach typically addresses the aleatory uncertainty in the design variable as a random variable. Ouyang et al. [10] proposed a novel loss function approach which takes the model parameter uncertainty into account through a Bayesian probabilistic approach to achieve a better quality. Youn et al. [11] presented a performance measure approach and a hybrid mean value for the reliability-based design optimization in the crashworthiness of the vehicle side impact. Du et al. [12] developed a sequential optimization and a reliability assessment to improve the efficiency of the probabilistic optimization. Stefanou [13] provided a state-of-the-art review of past and recent developments in the stochastic finite element method.

The interval approach typically addresses the epistemic uncertainty in the design variable as an interval variable. Recently, the interval approach for the epistemic uncertainty has achieved extensive concerns and several epis- temic uncertainty analysis approaches have been reported. Kang et al. [14] reviewed five common non-probabilistic reliability approaches and pointed out two indispensable features for a qualified reliability approach under the epistemic uncertainty. Zhang et al. [15] developed an interval Monte Carlo method which incorporated the interval analysis into the simulation process. This approach can easily obtain an accurately reliability assessment when the interval finite element method is used to model the ranges of the structural responses. Fujita et al. [16] proposed an enhanced approach for the robustness evaluation of an uncertain structure by the interval analysis with the secondorder Taylor series expansion. Beck et al. [17] presented a robust formulation of the structure risk optimization, which was less sensitive to the epistemic uncertainty.

Motivated by the present studies, we propose an RSMbased HRDO approach for the CPCM via integrating the probabilistic approach with the interval approach to cope with the mixed uncertainties in the hybrid design optimization. From the existing studies, we can know that the proposed RSM-based HRDO approach in the compliant mechanism filed is promising, yet mostly unexplored.

The format of this paper is structured as follows. Section 2 reviews the RSM modeling technique. Section 3 develops an RSM-based HRDO approach which is formulated based on the hybrid robust optimization approach and the RSM modeling technique. Section 4 illustrates the effectiveness of the proposed approach through a bridgetype amplification mechanism. Conclusions and further research are provided in Section 5.

\section{Modeling technique: RSM}

The RSM modeling technique plays a critical role for constructing an accurate empirical model between the input and the output. In practice, the quality characteristic of the CPCM is difficult to obtain by means of physical mechanisms. To overcome this issue, a major task is to identify the input-output functional model that relates to design variables and the quality characteristic. In this section, we will give a brief description of the RSM modeling technique.

\subsection{Response surface approximation}

The RSM modeling technique was introduced in [18], as an estimation of the true model. Thus, the RSM modeling technique is frequently used in the process modeling to replace the complex black-box function for the purpose of the robust design optimization. In the context of the RSM modeling technique, let an input vector $\boldsymbol{x}=$ $\left[x_{1}, x_{2}, \ldots, x_{k}\right]^{\prime}$ represents the design variables. An output vector $\boldsymbol{y}$ represents the quality characteristic. Then, the 
quality characteristic $\boldsymbol{y}$ can be approximated by the RSM approximation $\widehat{\boldsymbol{y}}$ with $k$ independent design variables. To determine a critical point for the polynomial function, a higher order polynomial such as the quadratic RSM model can be represented as follows [19-21]:

$\boldsymbol{y}(\boldsymbol{x})=\beta_{0}+\sum_{i=1}^{k} \beta_{i} x_{i}+\sum_{i=1}^{k} \beta_{i i} x_{i}^{2}+\sum_{i=1}^{k} \sum_{j \geqslant i}^{k} \beta_{i j} x_{i} x_{j}+\varepsilon$.

Define

$$
\boldsymbol{D}(\boldsymbol{x})=\left[1, x_{1}, \ldots, x_{k}, x_{1}^{2}, \ldots, x_{k}^{2}, x_{1} x_{2}, \ldots, x_{k-1} x_{k}\right]^{\prime}
$$

and

$$
\boldsymbol{\beta}=\left[\beta_{0}, \beta_{1}, \ldots, \beta_{k}, \beta_{11}, \ldots, \beta_{k k}, \beta_{12}, \ldots, \beta_{k-1, k}\right]^{\prime}
$$

then (2) becomes

$$
\boldsymbol{y}(\boldsymbol{x})=\boldsymbol{D}(\boldsymbol{x})^{\prime} \boldsymbol{\beta} .
$$

Suppose $Y$ is the observed quality characteristic in the experimental runs, then

$$
Y=\boldsymbol{y}(\boldsymbol{x})+\varepsilon
$$

where $\varepsilon$ is the experimental error term with $\varepsilon \sim N\left(0, \sigma^{2}\right)$ and $E(Y \mid \boldsymbol{x})=\boldsymbol{y}(\boldsymbol{x})$.

By using the least square estimates, the RSM model can be obtained as follows:

$$
\widehat{y}(\boldsymbol{x})=\boldsymbol{D}(\boldsymbol{x})^{\prime} \widehat{\boldsymbol{\beta}} .
$$

In the interest of determining unknown model parameters, the least square method is used to generate estimators. According to Ouyang et al. [22], the least square estimates $\widehat{\boldsymbol{\beta}}$ can be obtained as follows:

$$
\widehat{\boldsymbol{\beta}}=\left[\boldsymbol{X}^{\prime} \boldsymbol{X}\right]^{-1} \boldsymbol{X}^{\prime} \boldsymbol{y}
$$

where $\boldsymbol{X}$ is a data matrix.

Then, the predictive value at location $\boldsymbol{x}_{i}$ is given by

$$
\widehat{\boldsymbol{y}}(\boldsymbol{x})=\boldsymbol{D}\left(\boldsymbol{x}_{i}\right) \widehat{\boldsymbol{\beta}} .
$$

\subsection{Regression model accuracy metrics}

To ensure that the RSM model can achieve sufficient accuracy, three statistic criteria, i.e., relative mean squared error (MSE), average absolute error (AAE) and maximum absolute error (MAE) are used to verify the accuracy of the RSM model $[23,24]$. The lower value of the statistic criteria is, the better predictive accuracy of the RSM model is. Let the vector $\left(y_{1}, \ldots, y_{m}\right)^{\prime}$ be the actual values of the quality characteristic with mean $\bar{y}_{m}$ for the test data $\left(x_{1}, \ldots, x_{m}\right)^{\prime}$. The vector $\left(\widehat{y}_{1}, \ldots, \widehat{y}_{m}\right)^{\prime}$ denotes a vector of the values of the predicted quality characteristic. The statistic criteria can be expressed by

$$
\begin{aligned}
M S E & =\frac{\sum_{i=1}^{m}\left(y_{i}-\widehat{y}_{i}\right)^{2}}{m} \\
A A E & =\frac{\sum_{i=1}^{m}\left|y_{i}-\widehat{y}_{i}\right|}{m} \\
M A E & =\max \left|y_{i}-\widehat{y}_{i}\right|
\end{aligned}
$$

where $m$ is the number of experimental runs.

\section{Proposed HRDO approach for CPCM}

In this section, we propose an HRDO approach which considers the mixed uncertainties in the robust design optimization. RSM models are adopted to the assessment of the robustness and the robust design optimization.

\subsection{Uncertainty quantification in HRDO}

Most CPCMs are subjected to the mixed uncertainties, which can lead to the variation in the quality characteristic. Therefore, the robust assessment should measure the mixed uncertainties in the robust design optimization. Mathematically, the epistemic uncertainty is assessed by the width of an interval $\delta \sigma$ [25], since the limited information of a design variable only knows the range of the design variable lies. Meanwhile, the aleatory uncertainty is measured by the standard deviation $\sigma_{f_{\mathrm{RSM}}}$ and the mean value $\mu_{f_{\mathrm{RSM}}}$ [26], that the design variable is assumed to be a specific probability distribution.

Suppose a design variable $\boldsymbol{a}$ is an interval variable. Let $\boldsymbol{a}^{\boldsymbol{L}}$ and $\boldsymbol{a}^{\boldsymbol{U}}$ be the lower and upper bounds of the interval variable. The functional relationship between design variables and the quality characteristic can be in the form of $f_{\mathrm{RSM}}=f_{\mathrm{RSM}}(\boldsymbol{a})$. If there is only epistemic uncertainty in design variables, according to the interval theory, the width of the interval $\delta f_{\mathrm{RSM}}$ and the midpoint $\bar{f}_{\mathrm{RSM}}$ can be written as follows:

$$
\delta f_{\mathrm{RSM}}=f_{\mathrm{RSM}}^{U}-f_{\mathrm{RSM}}^{L}
$$

and

$$
\bar{f}_{\mathrm{RSM}}=\frac{\left(f_{\mathrm{RSM}}^{U}+f_{\mathrm{RSM}}^{L}\right)}{2} .
$$

Let the RSM modeling technique relate the quality characteristic $f_{\mathrm{RSM}}$ and a vector of random variables $\boldsymbol{x}=$ $\left[x_{1}, x_{2}, \ldots, x_{e}\right]$ with the form of $f_{\mathrm{RSM}}=f_{\mathrm{RSM}}(\boldsymbol{x})$. If there is only aleatory uncertainty in design variables, according to the probabilistic theory, the variance $\sigma_{f_{\mathrm{RSM}}}^{2}$ and the mean $\mu_{f_{\mathrm{RSM}}}$ of $f_{\mathrm{RSM}}$ can be acquired as follows:

$$
\mu_{f_{\mathrm{RSM}}}=E\left[f_{\mathrm{RSM}}(\boldsymbol{x})\right]=\int_{-\infty}^{\infty} f_{\mathrm{RSM}}(\boldsymbol{x}) \rho(\boldsymbol{x}) \mathrm{d} \boldsymbol{x}
$$




$$
\begin{gathered}
\sigma_{f_{\mathrm{RSM}}}^{2}=E\left[\left(f_{\mathrm{RSM}}(\boldsymbol{x})-\mu_{f_{\mathrm{RSM}}}\right)^{2}\right]= \\
\int_{-\infty}^{\infty}\left[f_{\mathrm{RSM}}(\boldsymbol{x})-\mu_{f_{\mathrm{RSM}}}\right]^{2} \rho(\boldsymbol{x}) \mathrm{d} \boldsymbol{x}
\end{gathered}
$$

where $\rho(\boldsymbol{x})$ is the joint probability density function.

It is worth noting that analytical solutions of both the variance $\sigma_{f_{\mathrm{RSM}}}^{2}$ and the mean $\mu_{f_{\mathrm{RSM}}}$ are difficult to acquire in high dimension. For this reason, the Monte Carlo simulation (MCS) is employed to assess the robustness in the robust design optimization.

\subsection{Robust assessment with mixed uncertainties}

As a result of the existence of both interval variables $\boldsymbol{a}=$ $\left(a_{1}, a_{2}, \ldots, a_{c}\right)$ and random variables $\boldsymbol{x}=\left(x_{1}, x_{2}, \ldots\right.$, $x_{e}$ ) in practical engineering issues, the mean value of the quality characteristic can be characterized on the basis of intervals for each RSM model. Therefore, the average mean value of the quality characteristic $f_{\mathrm{RSM}}(\boldsymbol{a}, \boldsymbol{x})$ can be computed by

$$
\bar{\mu}_{f_{\mathrm{RSM}}}=\frac{\left(\mu_{f_{\mathrm{RSM}}}^{\max }+\mu_{f_{\mathrm{RSM}}}^{\min }\right)}{2}
$$

where $\mu_{f_{\mathrm{RSM}}}^{\min }$ and $\mu_{f_{\mathrm{RSM}}}^{\max }$ are the minimum average value and the maximum average value for an interval, respectively.

With the purpose of quantifying the effect of randomness on the quality characteristic $f_{\mathrm{RSM}}(\boldsymbol{a}, \boldsymbol{x})$, the standard deviation $\sigma_{f_{\mathrm{RSM}}}$ is also bounded by the minimum standard deviation $\sigma_{f_{\mathrm{RSM}}}^{\min }$ and the maximum standard deviation $\sigma_{f_{\mathrm{RSM}}}^{\max }$. Therefore, the average standard deviation is given by

$$
\bar{\sigma}_{f_{\mathrm{RSM}}}=\frac{\left(\sigma_{f_{\mathrm{RSM}}}^{\max }+\sigma_{f_{\mathrm{RSM}}}^{\min }\right)}{2} .
$$

To quantify the effect of interval variables on the quality characteristic $f_{\mathrm{RSM}}(\boldsymbol{a}, \boldsymbol{x})$, the standard deviation difference $\delta \sigma_{f_{\mathrm{RSM}}}$ is used [27], which can be written as follows:

$$
\delta \sigma_{f_{\mathrm{RSM}}}=\sigma_{f_{\mathrm{RSM}}}^{\max }-\sigma_{f_{\mathrm{RSM}}}^{\min } .
$$

Overall, the mixed uncertainties of the quality characteristic can be quantified in terms of a distribution curve at each interval of the quality characteristic $f_{\mathrm{RSM}}(\boldsymbol{a}, \boldsymbol{x})$. That is to say, the interval at each probability level can illustrate the effect of epistemic uncertainty on the quality characteristic $f_{\mathrm{RSM}}(\boldsymbol{a}, \boldsymbol{x})$. It should be noted that both smaller values of $\delta \sigma_{f_{\mathrm{RSM}}}$ and $\bar{\sigma}_{f_{\mathrm{RSM}}}$ are desired to acquire the robust optimal solutions.

\subsection{Proposed RSM-based HRDO approach}

As reviewed previously, the prior design optimization mainly focuses on the traditional deterministic design op- timization approach without considering the mixed uncertainties, which can be generally formulated as follows:

$$
\left\{\begin{array}{c}
\underset{\boldsymbol{d}}{\operatorname{Min}} \quad Z=f_{\mathrm{RSM}}^{q}(\boldsymbol{d}), \quad q=1,2, \ldots, S \\
\text { s.t }\left\{\begin{array}{l}
g_{\mathrm{RSM}}^{i}(\boldsymbol{d}) \leqslant 0, \quad i=1,2, \ldots, L \\
h_{\mathrm{RSM}}^{j}(\boldsymbol{d})=0, \quad j=1,2, \ldots, I \\
\boldsymbol{d}_{v}^{L} \leqslant \boldsymbol{d} \leqslant \boldsymbol{d}_{v}^{U}, \quad v=1,2, \ldots, O \\
\boldsymbol{d}=\left[d_{1}, d_{2}, \ldots, d_{H}\right]^{\mathrm{T}}
\end{array}\right.
\end{array}\right.
$$

where $f_{\mathrm{RSM}}^{q}(q=1,2, \ldots, S)$ is the $q$ th quality characteristic. $g_{\mathrm{RSM}}^{i}(i=1,2, \ldots, L)$ is the $i$ th inequality constraint function. $h_{\mathrm{RSM}}^{j}(j=1,2, \ldots, I)$ is the $j$ th equality constraint function. $\boldsymbol{d}_{v}^{L}$ and $\boldsymbol{d}_{v}^{U}$ are the lower and upper bounds of the $v$ th design parameter, respectively. Obviously, (17) neglects the variations induced by the mixed uncertainties. It typically inclines to pushing the optimal design at one or more constraints such that the mixed uncertainties of design variables may make the traditional deterministic optimal design fall into the infeasible design.

To investigate the variation of the constraint caused by random design variables, Koch et al. [28] adopted a worstcase scenario to instead of the expected value of the response for the constraint, since it is more conservative than the probabilistic constraint. The constraint can be expressed as follows:

$$
\mu_{g_{\mathrm{RSM}}^{i}}^{\max }+\eta \sigma_{g_{\mathrm{RSM}}^{i}}^{\max } \leqslant 0, \quad i=1,2, \ldots, L
$$

where $\mu_{g_{\mathrm{RSM}}^{i}}^{\max }$ and $\sigma_{g_{\mathrm{RSM}}^{i}}^{\max }$ are the $i$ th maximum average value and the $i$ th maximum standard deviation of the $i$ th constraint, respectively. $\eta$ is the Sigma level. $\Phi(\eta)$ is the probability of confidence of the constraint satisfaction, which means the standard normal cumulative distribution function of the target reliability index (i.e., Sigma level).

Then, the RSM-based HRDO approach is proposed via considering random variables and interval variables in terms of the probabilistic theory and the interval theory. Then, the RSM-based HRDO approach can be formulated as follows:

$\left\{\begin{array}{l}\underset{\boldsymbol{d}}{\operatorname{Min}} \quad f_{\mathrm{RSM}}(\boldsymbol{\mu}, \boldsymbol{a})=w_{1} \bar{\mu}_{f_{\mathrm{RSM}}}+w_{2} \frac{\bar{\sigma}_{f_{\mathrm{RSM}}}}{\bar{\sigma}_{f_{\mathrm{RSM}}}^{*}}+w_{3} \frac{\delta \sigma_{f_{\mathrm{RSM}}}}{\delta \sigma_{f_{\mathrm{RSM}}^{*}}} \\ \text { s.t }\left\{\begin{array}{l}\boldsymbol{\mu}_{g_{\mathrm{RSM}}^{i}}^{\max }+\eta \boldsymbol{\sigma}_{g_{\mathrm{RSM}}^{i}}^{\max } \leqslant 0, \quad i=1,2, \ldots, L \\ h_{\mathrm{RSM}}^{i}(\boldsymbol{\mu}, \boldsymbol{a})=0, \quad j=1,2, \ldots, I \\ \boldsymbol{\mu}_{r}^{L} \leqslant \boldsymbol{\mu}_{r} \leqslant \boldsymbol{\mu}_{r}^{U}, \quad r=1,2, \ldots, c \\ \overline{\boldsymbol{a}}_{z}^{L} \leqslant \overline{\boldsymbol{a}}_{z} \leqslant \overline{\boldsymbol{a}}_{z}^{U}, \quad z=1,2, \ldots, e\end{array}\right.\end{array}\right.$

where $\boldsymbol{\mu}_{r}$ is the average value of the $r$ th random variable. $\boldsymbol{\mu}_{r}^{L}$ and $\boldsymbol{\mu}_{r}^{U}$ are the lower and upper bounds of the $r$ th random variable. $\overline{\boldsymbol{a}}_{z}$ is the average value of the interval variable. $\overline{\boldsymbol{a}}_{z}^{L}$ and $\overline{\boldsymbol{a}}_{z}^{U}$ are the given lower and upper bounds of the $z$ th interval variable. $\bar{\mu}_{f_{\mathrm{RSM}}}$ is the average value of the 
objective function. $\bar{\sigma}_{f_{\mathrm{RSM}}}^{*}$ and $\delta \sigma_{f_{\mathrm{RSM}}}^{*}$ are the best achievable optimal solutions of $\bar{\sigma}_{f_{\mathrm{RSM}}}$ and $\delta \sigma_{f_{\mathrm{RSM}}}$, which are obtained with the traditional deterministic design optimization approach. $w_{i}$ is the weight coefficient.

To optimize the quality characteristic and analyze the mixed uncertainties in the robust design optimization, the dual-loop MCS is employed to evaluate $\delta \sigma_{f_{\mathrm{RSM}}}, \bar{\mu}_{f_{\mathrm{RSM}}}$ and $\bar{\sigma}_{f_{\mathrm{RSM}}}$ [29]. In the outer loop, a number of small segments $N_{i}$ intervals are generated. Then, each combination of the intervals is simulated. In the inner loop, the robustness of the quality characteristic is conducted. For clarification, a general flowchart of the dual-loop MCS procedure is shown in Fig. 1.

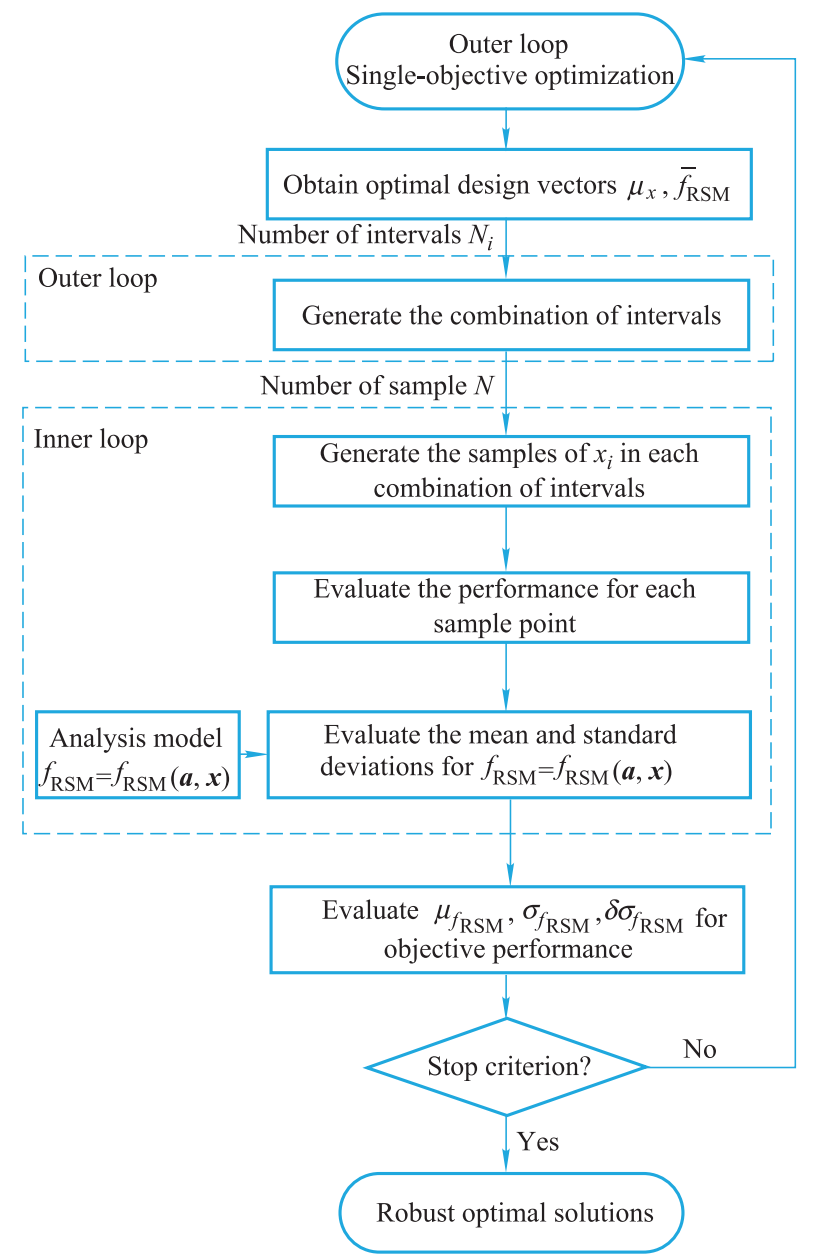

Fig. 1 Dual-loop MCS procedure

\subsection{Flowchart and procedure of proposed approach}

For clarification, a flowchart of the proposed approach is presented in Fig. 2. The primarily steps of the proposed approach can be summarized as follows:

Step 1 Determine the mixed uncertainties issue in the robust design optimization for the CPCM.
Step 2 Identify design variables, constraint, quality characteristic and then arrange the experimental runs according to the central composite design (CCD).

Step 3 Collect the experimental data via conducting the finite element (FE) simulation.

Step 4 Construct the objective and the constraint functions by means of the RSM modeling technique, and then evaluate the accuracy of RSM models. If the accuracy is close to the criteria, then end; otherwise, go to Step 2.

Step 5 Propose an RSM-based HRDO approach for the CPCM by considering the mixed uncertainties.

Step 6 Implement the dual-loop MCS to calculate $\bar{\mu}_{f_{\mathrm{RSM}}}, \bar{\sigma}_{f_{\mathrm{RSM}}}$ and $\delta \sigma_{f_{\mathrm{RSM}}}$, and then obtain robust optimal solutions.

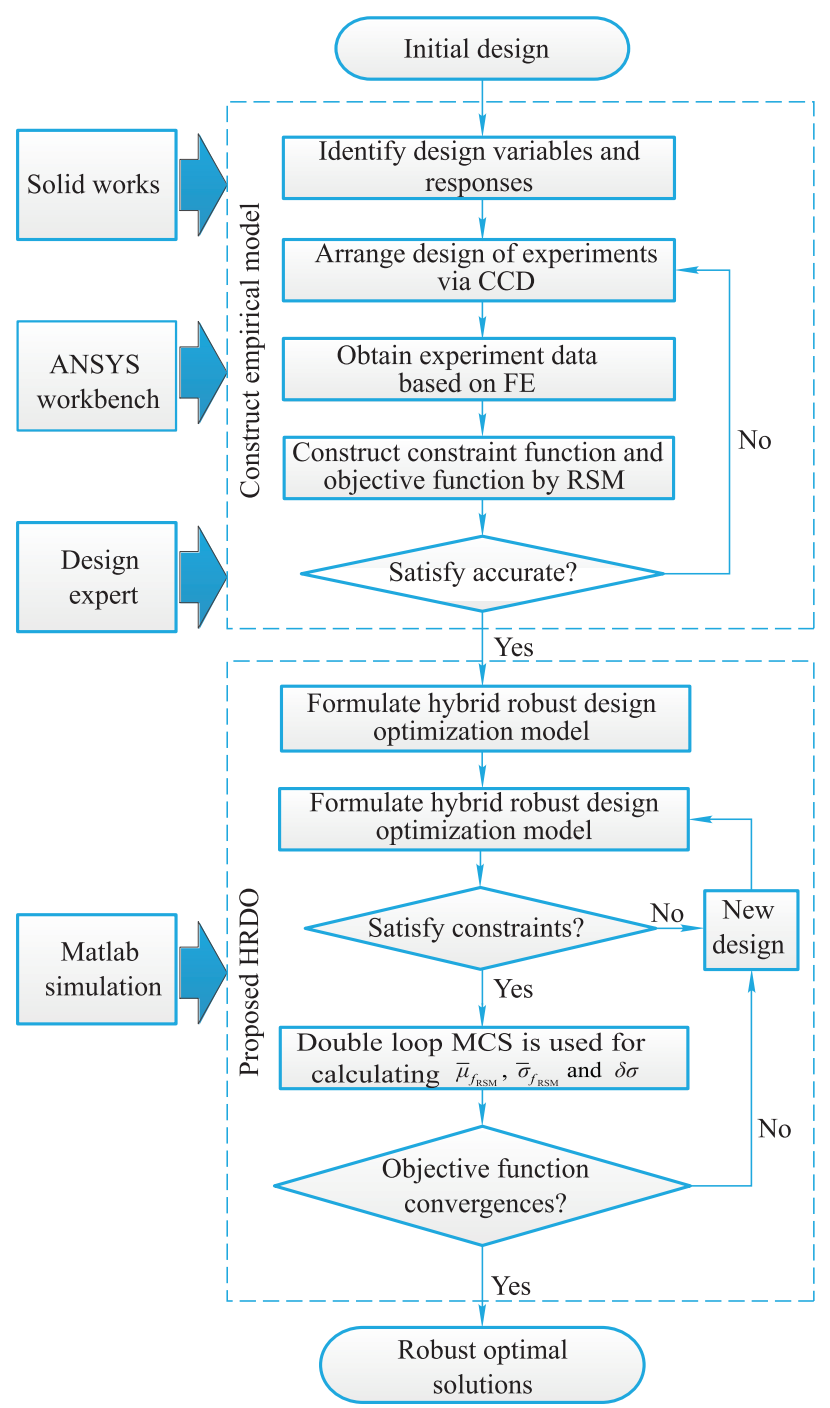

Fig. 2 Flowchart of the proposed approach

\section{Case study: bridge-type amplification mechanism}

In this section, a bridge-type amplification mechanism is 
employed to demonstrate the effectiveness of the proposed approach. We shall use the traditional deterministic design optimization approach for a comparison.

\subsection{Issue description}

A bridge-type amplification mechanism (displacement amplifier) is one of the typical CPCMs [30], which transfers an input displacement at one port to an output displacement at another port through the elastic body deformation. The bridge-type amplification mechanism has been widely employed in flexure-based micro-manipulators to enlarge the displacement of the end-effector, due to its compact size and large amplification ratio. For practical applications, the piezoelectric actuator (PZT) is adopted to drive the bridgetype amplification mechanism, which can provide an input displacement as a cycle loading. As a result of the symmetry of the bridge-type amplification mechanism, a quar- ter of the structure is selected to analyze the mechanical motions. In fact, the bridge-type amplification mechanism is required to magnify the input displacement $\Delta x$ from the PZT. When the bridge-type amplification mechanism is subjected to an input displacement $\Delta x$ by the PZT, the output end can be generated as an amplified $\Delta y$ output displacement. The amplification ratio is defined as $\frac{\Delta y}{\Delta x}$. The working principle is displayed in Fig. 3. The amplification ratio is mainly affected by four design variables which are the flexible hinge thickness $x_{1}$, the rigid beam length $x_{2}$, the flexible hinge length $x_{3}$ and the hinge distance $x_{4}$. The description of four design variables is exhibited in Table 1. In consequence of the mixed uncertainties, the design variables frequently occur variations, which could lead to the amplification ratio variability. The objective of this paper is to improve the quality of the amplification ratio via the proposed approach.

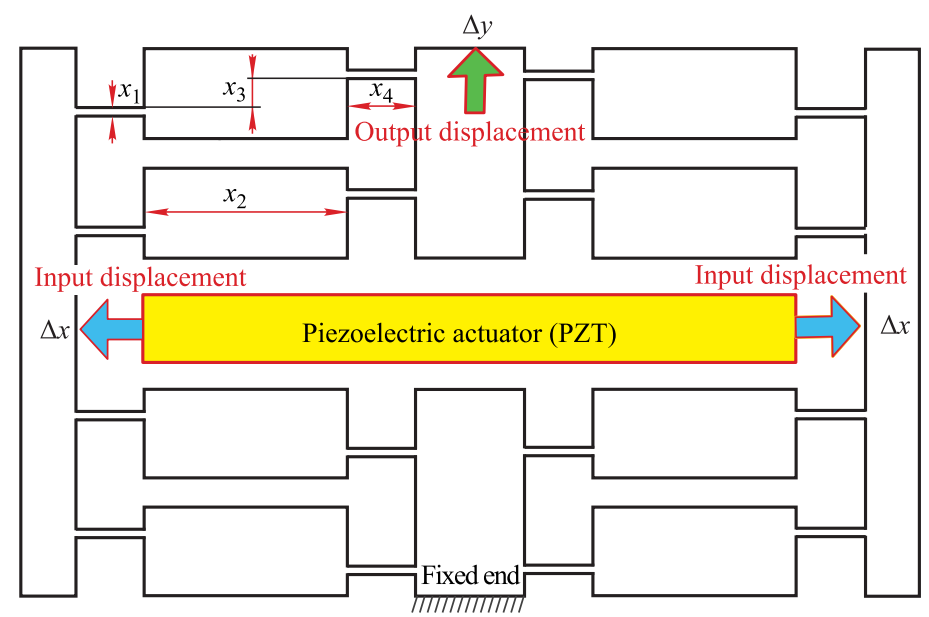

Fig. 3 Symmetrical bridge-type amplification mechanism

Table 1 Design variables in the bridge-type amplification mechanism

\begin{tabular}{ccccc}
\hline Variable & Represent & Unit & $\begin{array}{c}\text { Low } \\
\text { level }\end{array}$ & $\begin{array}{c}\text { High } \\
\text { level }\end{array}$ \\
\hline$x_{1}$ & Flexible hinge thickness & $\mathrm{mm}$ & 0.48 & 0.52 \\
$x_{2}$ & Rigid beam length & $\mathrm{mm}$ & 14.8 & 15.2 \\
$x_{3}$ & Hinge distance & $\mathrm{mm}$ & 1.8 & 2.2 \\
$x_{4}$ & Flexible hinge length & $\mathrm{mm}$ & 4.8 & 5.2 \\
\hline
\end{tabular}

\subsection{Design of experiments and construct RSM models}

Attributable to the true functional models for the quality characteristic is not easy to acquire by the theoretical derivation. Therefore, the RSM modeling technique is used to construct empirical models for the maximum equivalent stress and the amplification ratio, respectively. In this case, the CCD is employed to implement the experiments.

A CCD with four design variables is performed, resulting in 30 different experimental runs. Instead of conduct- ing the actual experiments in a laboratory, the FE simulation is employed to generate the experimental data for 30 different experimental runs. It should be noted that the polypropylene is selected for the material. The Young's modulus, the Poisson's ratio and the yield stress are $1.4 \mathrm{GPa}, 0.41$, and $34 \mathrm{MPa}$, respectively. Subsequently, the FE mesh division and the FE simulation for the bridgetype amplification mechanism are analyzed. The automatic method is utilized for meshing the model, and the minimum element size is selected as $1 \mathrm{~mm}$. It should be noted that the cycle loading condition is driven by providing input displacement $0.001 \mathrm{~mm}$ from the PZT. In the ANSYS workbench platform, the definition of the constraints and the meshed model for the bridge-type amplification mechanism is shown in Fig. 4. It can be seen in Fig. 4(a) that the external load is applied on the A surface of the fixed body as the fixed support, and the B surface and the $\mathrm{C}$ surface of two input bodies are displaced by $0.001 \mathrm{~mm}$, respectively. 


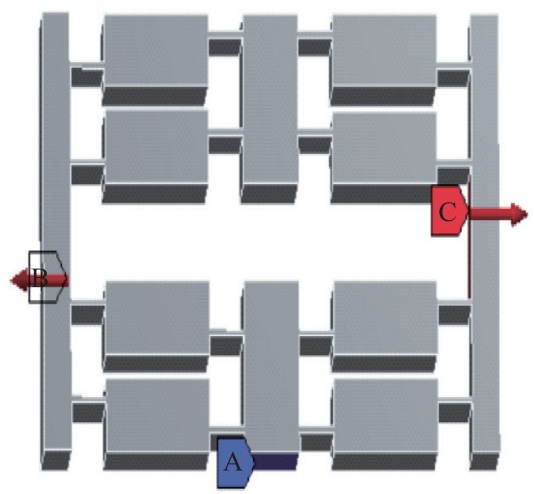

(a) Fixed and input displacement for the mechanism

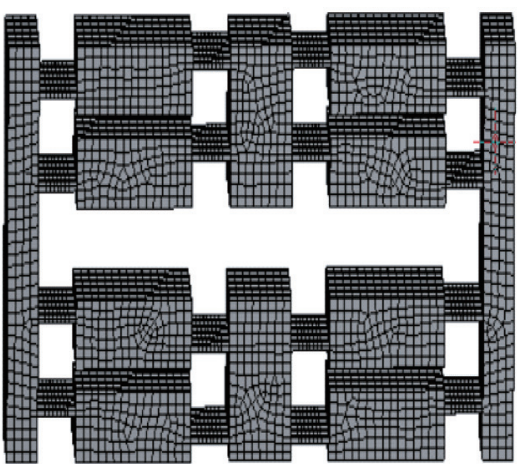

(b) Meshed model
: Fixed support;
: Displacement;
: Displacement2.

Fig. 4 Finite element model of the bridge-type amplification mechanism

According to the FE simulation, the experimental results of different experimental runs can be obtained. As an example, when the Group 12 experiment is conducted, the results (the amplification ratio $y_{\mathrm{RSM}}^{A}$ and the maximum equivalent stress $\left.g_{\mathrm{RSM}}^{\sigma_{\max }}\right)$ for the Group 12 experiment are obtained. Fig. 5 demonstrates the stress distribution for the bridge-type amplification mechanism of the Group 12. Therefore, different results for 30 experimental runs are obtained in Table 2.
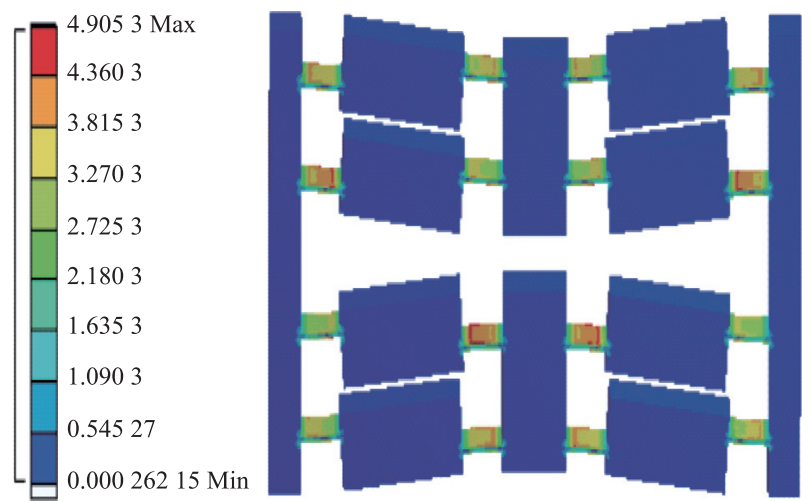

Fig. 5 Stress distribution of the bridge-type amplification mechanism

The experimental data is divided into two parts: one is the training data (Group 1 to Group 20), and the other is the testing data (Group 21 to Group 30). According to the training data, empirical models for the maximum equivalent stress $g_{\mathrm{RSM}}^{\sigma_{\max }}$ and the amplification ratio $y_{\mathrm{RSM}}^{A}$ are constructed via the RSM modeling technique. The $p$-value in the analysis of variance for the RSM models are 0.001 and 0.04 , respectively, which imply that the RSM models can be utilized in the subsequently robust design optimization.

$$
g_{\mathrm{RSM}}^{\sigma_{\max }}=923.8487-139.6180 x_{1}-77.9037 x_{2}-
$$

Table 2 Experimental results with 4 design variables and 2 responses

\begin{tabular}{ccccccc}
\hline Runs & $x_{1}$ & $x_{2}$ & $x_{3}$ & $x_{4}$ & $y_{\mathrm{RSM}}^{A}$ & $g_{\mathrm{RSM}}^{\sigma_{\max }}$ \\
\hline 1 & 1 & 1 & -1 & -1 & 16.1821 & 6.5981 \\
2 & -1 & -1 & -1 & 1 & 16.3299 & 6.6619 \\
3 & 1 & -1 & 1 & -1 & 13.8913 & 4.3341 \\
4 & 0 & 0 & 0 & 0 & 15.3945 & 4.9053 \\
5 & -1 & 1 & -1 & 1 & 16.6300 & 6.7480 \\
6 & -1 & 1 & 1 & 1 & 14.7593 & 4.0409 \\
7 & 1 & 1 & 1 & -1 & 14.1590 & 4.3874 \\
8 & 1 & -1 & -1 & -1 & 15.8099 & 7.3503 \\
9 & 0 & 0 & 0 & 0 & 15.3945 & 4.9053 \\
10 & 1 & -1 & 1 & 1 & 14.2146 & 3.9597 \\
11 & -1 & 1 & -1 & -1 & 16.3310 & 7.3634 \\
12 & 0 & 0 & 0 & 0 & 15.3945 & 4.9053 \\
13 & -1 & -1 & 1 & -1 & 14.1559 & 4.5163 \\
14 & 1 & -1 & -1 & 1 & 16.1781 & 6.2780 \\
15 & -1 & 1 & 1 & -1 & 14.4421 & 4.4799 \\
16 & 1 & 1 & 1 & 1 & 14.4843 & 4.0590 \\
17 & 1 & 1 & -1 & 1 & 16.4921 & 6.2914 \\
18 & -1 & -1 & -1 & -1 & 15.9651 & 7.7490 \\
19 & 0 & 0 & 0 & 0 & 15.3945 & 4.9053 \\
20 & -1 & -1 & 1 & 1 & 14.4749 & 4.0772 \\
21 & 0 & 0 & 0 & -2 & 15.0522 & 6.1774 \\
22 & -2 & 0 & 0 & 0 & 15.6032 & 5.3163 \\
23 & 0 & 0 & 0 & 2 & 15.6853 & 4.7568 \\
24 & 0 & 2 & 0 & 0 & 15.6923 & 5.2540 \\
25 & 0 & 0 & 0 & 0 & 15.3945 & 4.9053 \\
26 & 0 & 0 & -2 & 0 & 16.8268 & 8.8567 \\
27 & 0 & 0 & 2 & 0 & 13.2389 & 3.8294 \\
28 & 0 & -2 & 0 & 0 & 15.0868 & 5.2077 \\
29 & 0 & 0 & 0 & 0 & 15.3945 & 4.9053 \\
30 & 2 & 0 & 0 & 0 & 15.1649 & 4.7389 \\
\hline & & & & & &
\end{tabular}

$93.7624 x_{3}-76.9556 x_{4}-3.3474 x_{1} x_{2}+25.4744 x_{1} x_{3}+$ $7.7936 x_{1} x_{4}+1.7471 x_{2} x_{3}+2.0054 x_{2} x_{4}+2.3444 x_{3} x_{4}+$ $92.5507 x_{1}^{2}+2.1959 x_{2}^{2}+9.1471 x_{3}^{2}+3.6726 x_{4}^{2}$ $y_{\mathrm{RSM}}^{A}=-59.2331+22.6442 x_{1}+5.0860 x_{2}+14.5565 x_{3}+$ 
$5.8084 x_{4}-0.2032 x_{1} x_{2}-7.6410 x_{1} x_{3}+0.4213 x_{1} x_{4}-$

$0.3819 x_{2} x_{3}-0.1933 x_{2} x_{4}-0.0896 x_{3} x_{4}-11.7404 x_{1}^{2}-$

$$
0.0830 x_{2}^{2}-2.3126 x_{3}^{2}-0.2129 x_{4}^{2}
$$

To evaluate the accuracy of RSM models, the performance evaluation criteria ( $M S E, A A E$, and $M A E$ ) are adopted. The accurate assessments of RSM models are summarized in Table 3. As revealed in Table 3, the first two columns' values of the $M S E$ and $A A E$ are $0.0015,0.0059,0.0261$ and 0.0629 , respectively, which mean the high accuracy of RSM models. In addition, the last column' values of the $M A E$ are 0.0965 and 0.1302 , respectively, which indicate the small error in one region of the design space even though an excellent global accuracy represented by the $M S E$ and the $A A E$. Overall, the results in Table 3 manifest that RSM models can be considered as a substitute for the true functional models at the allowable accuracy.
Table 3 Accuracy assessment of RSM models

\begin{tabular}{cccc}
\hline Items & $M S E$ & $A A E$ & $M A E$ \\
\hline$y_{\mathrm{RSM}}^{A}$ & 0.0015 & 0.0261 & 0.0965 \\
$g_{\mathrm{RSM}}^{\sigma \max }$ & 0.0059 & 0.0629 & 0.1302 \\
Allowable value & $\leqslant 0.2$ & $\leqslant 0.2$ & $\leqslant 0.2$ \\
\hline
\end{tabular}

\subsection{Optimization results and further discussion}

In the robust design optimization, the uncertain structure parameters $\left(x_{1}, x_{2}, x_{4}\right)$ are assumed to be normally distributed as random variables according to the practical engineering experience. Since the uncertain parameter $x_{3}$ is obtained by the limited information, it is treated as an interval variable. The other structure parameters of the bridgetype amplification mechanism are considered as the deterministic parameters. Without loss of generality, the coefficient of variation and the interval variation are assumed $1 \%$ and $2.5 \%$ in the proposed approach, according to the engineering design experience. The design variables with the mixed uncertainties are shown in Table 4.

Table 4 Random variables and interval variable

\begin{tabular}{|c|c|c|c|c|c|}
\hline \multicolumn{4}{|c|}{ Random variable } & \multicolumn{2}{|r|}{ Interval variable } \\
\hline Variable & Mean & Standard deviation & Distribution & Variable & Interval \\
\hline$x_{1}$ & $\mu_{x_{1}}$ & $\sigma_{x_{1}}=0.01 \mu_{x_{1}}$ & Normal & & \\
\hline$x_{2}$ & $\mu_{x_{2}}$ & $\sigma_{x_{2}}=0.01 \mu_{x_{2}}$ & Normal & $x_{3}$ & $\left(x_{3}-0.025 x_{3}, x_{3}+0.025 x_{3}\right)$ \\
\hline$x_{4}$ & $\mu_{x_{4}}$ & $\sigma_{x_{4}}=0.01 \mu_{x_{4}}$ & Normal & & \\
\hline
\end{tabular}

The objective of the bridge-type amplification mechanism robust design optimization issue is to find the optimum designs that maximize the amplification ratio and satisfy an allocated reliability target with respect to the maximum equivalent stress, while accounting for the mixed uncertainties. The maximum stress generated $g_{\mathrm{RSM}}^{\sigma_{\max }}$ should not exceed the yield stress of the material, which is used as a six Sigma quality constraint. To improve the robustness of the bridge-type amplification mechanism, the proposed approach in (18) is adopted to formulate the optimization issue. Then, the robustness for the bridgetype amplification mechanism are quantified by $\bar{\sigma}_{f_{\mathrm{RSM}}}$ and $\delta \sigma_{f_{\mathrm{RSM}}}$. In the dual-loop MCS, 20000 independent samples for the random variables and 40 intervals for the interval variable are taken. The proposed robust design optimization is executed starting from the traditional deterministic optimal solutions. Then, the sequential quadratic programming algorithm is employed to perform the proposed robust design optimization.

To demonstrate the superiority of the proposed approach, the MCS is employed in the following confirmatory simulation. As aforesaid, the robustness of the quality characteristic is to calculate $\bar{\sigma}_{f_{\mathrm{RSM}}}$ and $\delta \sigma_{f_{\mathrm{RSM}}}$. To do so, the dual-loop MCS is used to calculate $\bar{\sigma}_{f_{\mathrm{RSM}}}$ and $\delta \sigma_{f_{\mathrm{RSM}}}$ by taking 40 intervals and 20000 independent samples. For each design variable, the interval variable is divided into 40 intervals $\left(N_{i}=40\right)$, and 20000 independent samples $(N=20000)$ are taken for three random variables. Since there is one interval variable $(P=1)$, the number of the objective function for robustness evaluations are thereby equal to $N_{i}^{P} \times N=40^{1} \times 20000=8 \times 10^{5}$ for the MCS. Then, optimal solutions are obtained in Table 5. It is interesting to see that two settings of design parameters from different approaches are fairly close to each other. However, the values of $\bar{\sigma}_{f_{\mathrm{RSM}}}$ and $\delta \sigma_{f_{\mathrm{RSM}}}$ contrast fairly markedly.

It can be seen in Table 5 that $\bar{\sigma}_{y_{\mathrm{RSM}}^{A}}=0.1811$ and $\delta \sigma_{y_{\mathrm{RSM}}^{A}}=0.0046$ from the proposed approach is smaller than their counterparts $\bar{\sigma}_{y_{\mathrm{RSM}}^{A}}=0.1807$ and $\delta \sigma_{y_{\mathrm{RSM}}^{A}}=$ 0.0034 from the traditional deterministic design optimization approach. Put it another way, the ranges of $\bar{\sigma}_{y_{\mathrm{RSM}}^{A}}$ and $\delta \sigma_{y_{\text {RSM }}^{A}}$ from the proposed approach are significantly narrower than those from the traditional deterministic design optimization approach. From the robustness point of view, the results demonstrate that the proposed approach is more robust than the traditional deterministic design optimization approach. The reason for this phenomenon derives that the proposed approach accounts for the mixed uncertain- 
ties in design variables. Moreover, the performance of the amplification ratio has a remarkable improvement which increases from 13.4243 times to 14.8974 times (an increase of about 1.4731 times).

Table 5 Comparison of the robustness assessment

\begin{tabular}{ccccc}
\hline \multicolumn{2}{c}{ Design variable } & & \multicolumn{2}{c}{ Optimal solution } \\
\cline { 1 - 2 } \cline { 5 - 5 } $\begin{array}{c}\text { Deterministic } \\
\text { approach }\end{array}$ & $\begin{array}{c}\text { Proposed } \\
\text { approach }\end{array}$ & & $\begin{array}{c}\text { Deterministic } \\
\text { solution }\end{array}$ & $\begin{array}{c}\text { Robust } \\
\text { solution }\end{array}$ \\
\hline$x_{1}$ & $\mu_{x_{1}}$ & & 0.4752 & 0.4701 \\
$x_{2}$ & $\mu_{x_{2}}$ & & 14.7885 & 14.7760 \\
$x_{3}$ & $x_{3}$ & & 5.1729 & 5.1827 \\
$x_{4}$ & $\mu_{x_{4}}$ & & 2.3883 & 2.3997 \\
$y_{\mathrm{RSM}}^{A}$ & $y_{\mathrm{RSM}}^{A}$ & & 13.4243 & 14.8974 \\
$\bar{\sigma}_{y_{\mathrm{RSM}}^{A}}$ & $\bar{\sigma}_{y_{\mathrm{RSM}}^{A}}$ & & 0.1811 & 0.1807 \\
$\delta \sigma_{y_{\mathrm{RSM}}^{A}}$ & $\delta \sigma_{y_{\mathrm{RSM}}^{A}}$ & & 0.0046 & 0.0034 \\
\hline
\end{tabular}

To better demonstrate the superiority of the proposed approach, a comparison of the families of the probability density function curves are displayed in Fig. 6. The narrow widths of the normal distribution curves illustrate the low sensitivities $\delta \sigma_{y_{\mathrm{RSM}}^{A}}$ and the small values $\bar{\sigma}_{y_{\mathrm{RSM}}^{A}}$.

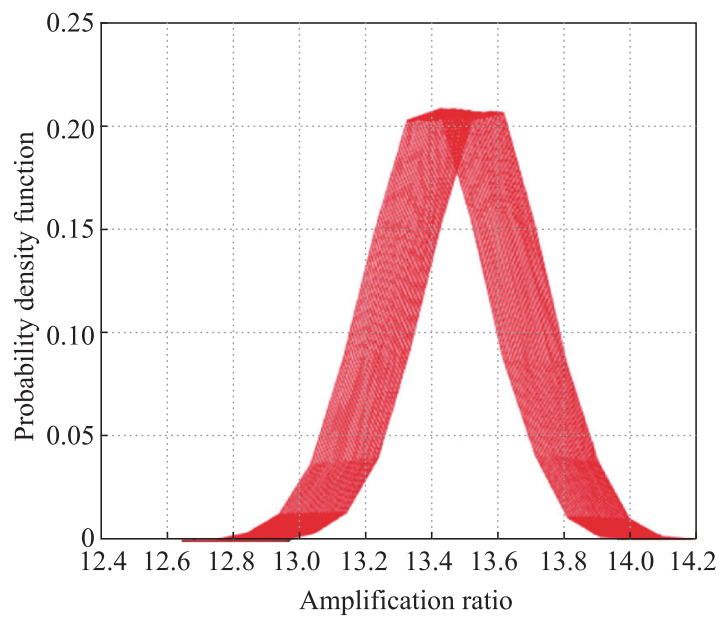

(a) Robust optimum design

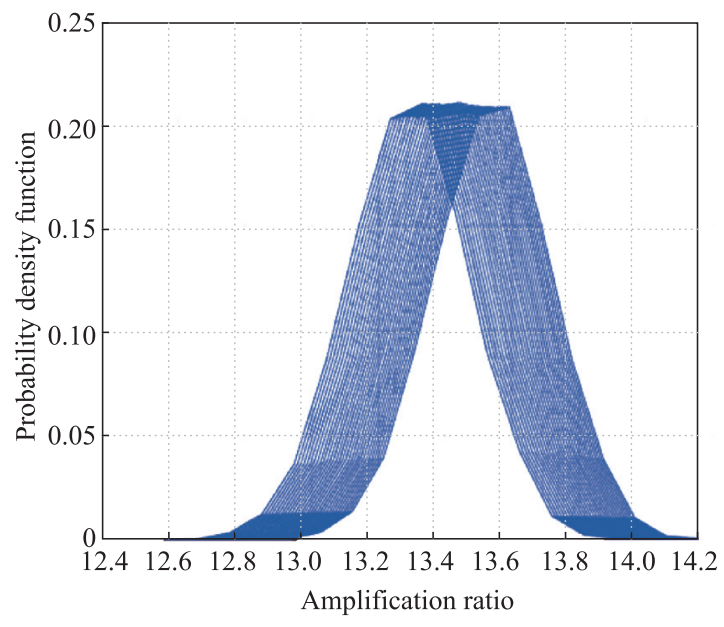

(b) Traditional deterministic optimum design

Fig. 6 Comparison of the family of distributions
Apparently, the widths of the normal distribution curves from the robust optimal solutions are narrower than the traditional deterministic optimal solutions counterparts. Thereby, the robustness of the proposed approach is much higher than that of the traditional deterministic design optimization approach.

To further validate the robustness of the proposed approach, the FE simulation is utilized to validate the two different optimal solutions via calculating the equivalent stress for the maximum values under the same working condition. Fig. 7 depicts a comparison of the contour plots of the maximum equivalent stress. It can be seen from Fig. 7 that the maximum equivalent stress decreases from $4.9053 \mathrm{MPa}$ to $3.7108 \mathrm{MPa}$. That is to say, the maximum equivalent stress reduces $26.39 \%$ compared to the initial design. However, the traditional deterministic optimal design can achieve $24.35 \%$ decrease in terms of the maximum equivalent stress compared with the initial design under the same condition. The foremost reason for this phenomenon is that the robust optimal design has a great bearing capacity to the carry larger load. As seen from Fig. 7, the maximum equivalent stress of the traditional deterministic optimal design is about $3.7184 \mathrm{MPa}$, and the stress state of the robust optimal design is relatively lower than the traditional deterministic optimal design. From the structure reliability point of view, the smaller the peak stress generates, the higher reliability the state keeps. Although there is a reduction of the maximum equivalent stress from the traditional deterministic optimal design, it does not account for the mixed uncertainties in the design stage. In other words, the traditional deterministic optimal design may increase the risk of structure failure.

From above-mentioned results, it can be observed that the quality characteristic has a significant enhancement. These results imply that the proposed approach is fairly effective in enhancing the robustness and the performance. Overall, the proposed approach is considered promising for the CPCM.

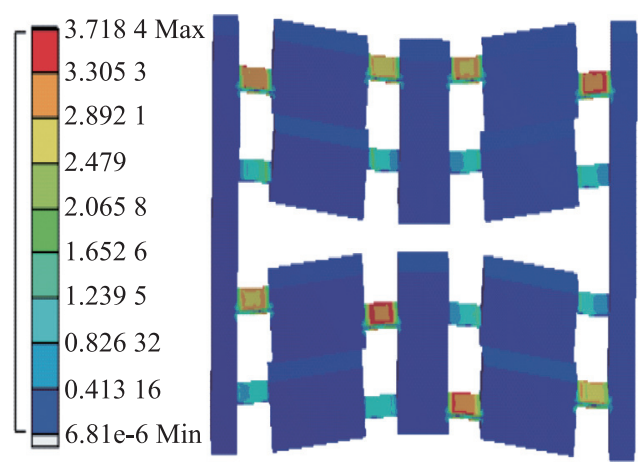

(a) Traditional deterministic optimum design 


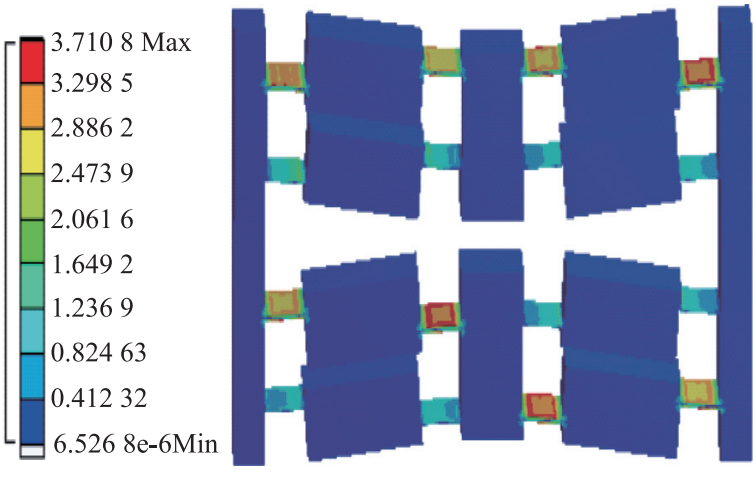

(b) Robust optimum design

Fig. 7 Comparison of stress distribution

\section{Conclusions}

An RSM-based HRDO approach for the CPCM is proposed to consider a series of issues including the mixed uncertainties, the quality, and the reliability in the robust design optimization. This paper makes a foremost contribution for the CPCM: an integrated HRDO approach considering the mixed uncertainties and the RSM modeling technique in a unified framework. A bridge-type amplification mechanism is used to demonstrate the effectiveness of the proposed approach. From the comparison results, the proposed approach is a good alternative for the practitioners as it can provide a robust optimization scheme. Furthermore, the comparison results also manifest that the proposed approach can not only reduce the variation in the quality characteristic, but also enhance the performance.

A challenging work for the further research is to improve the evaluate efficiency of the mean and the standard deviation of the quality characteristic. If the number of random samples and intervals are not large enough, the extreme values of the results would be missed. Furthermore, since empirical models derived from experiments are widely used in the engineering issues, a wellestablished empirical modeling technique thereby plays a key role in constructing the underlying mechanistic model of the CPCM. Thus, the further research also may extend the proposed approach to the highly nonlinear underlying mechanistic model. Consequently, more efforts are needed to improve the computational efficiency and construct a well-established empirical model.

\section{References}

[1] HOWELL L L, MAGLEBY S P, OLSEN B M. Handbook of compliant mechanisms. New York: Wiley, 2013.

[2] ZHAO Q H, CHEN X K, MA Z D, et al. A comparison of deterministic, reliability-based topology optimization under uncertainties. Acta Mechanica Solida Sinica, 2016, 29(1): $31-$ 45.

[3] WANG X J, GENG X Y, WANG L, et al. Motion error based robust topology optimization for compliant mechanisms under material dispersion and uncertain forces. Structural \& Multidisciplinary Optimization, 2017, 547(6): 2161-2175.

[4] LI G J, LU Z Z, LI L Y, et al. Aleatory and epistemic uncertainties analysis based on non-probabilistic reliability and its kriging solution. Applied Mathematical Modelling, 2016, 40(9-10): $5703-5716$.

[5] WANG X J, GENG X Y, WANG L, et al. Motion error based robust topology optimization for compliant mechanisms under material dispersion and uncertain forces. Structural \& Multidisciplinary Optimization, 2018, 57: $2161-2175$.

[6] WITTWER J W, BAKER M S, HOWELL L L. Robust design and model validation of nonlinear compliant micromechanisms. Journal of Microelectromechanical Systems, 2005, 15(1): $33-41$.

[7] LAZAROV B S, SCHEVENELS M, SIGMUND O. Robust design of large-displacement compliant mechanisms. Mechanical Sciences, 2011, 2(2): 175-182.

[8] GARCIA-LOPEZ N P, SANCHEZ-SILVA M, MEDAGLIA A $\mathrm{L}$, et al. An improved robust topology optimization approach using multiobjective evolutionary algorithms. Computers \& Structures, 2013, 125: 1-10.

[9] DAO T P, HUANG S C, CHAU N L. Robust parameter design for a compliant microgripper based on hybrid Taguchidifferential evolution algorithm. Microsystem Technologies, 2018, 24: 1461 - 1477.

[10] OUYANG L H, MA Y Z, CHEN J X, et al. Robust optimisation of ND: YLF laser beam micro-drilling process using Bayesian probabilistic approach. International Journal of Production Research, 2016, 54(2): 1-16.

[11] YOUN B D, CHOI K K, YANG R J, et al. Reliability-based design optimization for crashworthiness of vehicle side impact. Structural \& Multidisciplinary Optimization, 2004, 26(3-4): $272-283$.

[12] DU X P, CHEN W. Sequential optimization and reliability assessment method for efficient probabilistic design. Journal of Mechanical Design, 2003, 126(2): 871-880.

[13] STEFANOU G. The stochastic finite element method: past, present and future. Computer Methods in Applied Mechanics \& Engineering, 2009, 198(9): $1031-1051$.

[14] KANG R, ZHANG Q Y, ZENG Z G, et al. Measuring reliability under epistemic uncertainty: review on non-probabilistic reliability metrics. Chinese Journal of Aeronautics, 2016, 29(3): $571-579$.

[15] ZHANG H, MULLEN R L, MUHANNA R L. Interval Monte Carlo methods for structural reliability. Structural Safety, 2010, 32(3): $183-190$.

[16] FUJITA K, TAKEWAKI I. An efficient methodology for robustness evaluation by advanced interval analysis using updated second-order Taylor series expansion. Engineering Structures, 2011, 33(12): 3299-3310.

[17] BECK A T, GOMES W J S, BAZAN F A V. On the robustness of structural risk optimization with respect to epistemic uncertainties. Mecnica Computacional, 2012, 2(1): 1-19.

[18] BOX G E P, HUNTER W G, HUNTER J S. Statistics for experimenters: an introduction to design, data analysis and model building. New York: Wiley, 1978.

[19] KIM K J, LIN D K J. Optimization of multiple responses considering both location and dispersion effects. European Journal of Operational Research, 2006, 169(1): 133-145.

[20] HE Z, ZHU P F, PARK S H. A robust desirability function method for multi-response surface optimization considering model uncertainty. European Journal of Operational Research, 2012, 221(1): $241-247$.

[21] CHO B R, PARK C. Robust design modeling and optimiza- 
tion with unbalanced data. Computers \& Industrial Engineering, 2005, 48(2): $173-180$.

[22] OUYANG L H, MA Y Z, BYUN J H, et al. A prediction region-based approach to model uncertainty for multi-response optimization. Quality \& Reliability Engineering International, 2016, 32(3): 783-794.

[23] JIN R C, CHEN W, SIMPSON T W. Comparative studies of metamodelling techniques under multiple modelling criteria. Structural \& Multidisciplinary Optimization, 2001, 23(1): 1 13.

[24] OUYANG L H, ZHOU D Q, MA Y Z, et al. Ensemble modeling based on 0-1 programming in micro-manufacturing process. Computers \& Industrial Engineering, 2018, 123: 242 253.

[25] WU W D, RAO S S. Interval approach for the modeling of tolerances and clearances in mechanism analysis. Journal of Mechanical Design, 2004, 126(4): 581-592.

[26] HUANG B Q, DU X P. Analytical robustness assessment for robust design. Structural \& Multidisciplinary Optimization, 2007, 34(2): 123 - 137.

[27] DU X P, VENIGELLA P K, LIU D S. Robust mechanism synthesis with random and interval variables. Mechanism \& Machine Theory, 2009, 44(7): 1321 - 1337.

[28] KOCH P N, YANG R J, GU L. Design for six Sigma through robust optimization. Structural \& Multidisciplinary Optimization, 2004, 26(3-4): 235-248.

[29] DU X P, VENIGELLA P K, LIU D S. Robust mechanism synthesis with random and interval variables. Mechanism \& Machine Theory, 2009, 44(7): $1321-1337$.

[30] WU H, ZHANG X M, GAN J Q, et al. High-precision displacement measurement method for three degrees of freedomcompliant mechanisms based on computer micro-vision. Applied Optics, 2016, 55(10): $2594-2600$.

\section{Biographies}

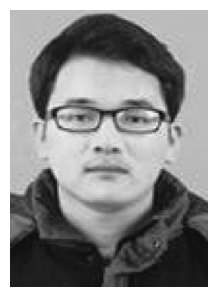

WAN Liangqi was born in 1991. He received his M.S. degree from Jiangxi University of Science and Technology in 2016. Now, he is a Ph.D. student at College of Economics and Management, Nanjing University of Aeronautics and Astronautics. He is also a joint Ph.D. student (2018.092019.09) in the Reliability Research Laboratory, University of Alberta. His research interests are reliability-based design optimization and robust design optimization.

E-mail: wanliangqi@nuaa.edu.cn

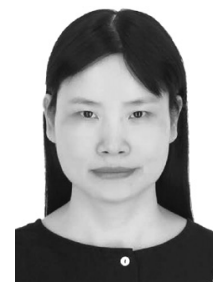

CHEN Hongzhuan was born in 1977. She received her M.S. degree from Shandong University of Science and Technology in 2002 and her Ph.D. degree from Hohai University in 2005. Now, she is a professor at College of Economics and Management, Nanjing University of Aeronautics and Astronautics. Her research interests are quality management and quality engineering.

E-mail: hongzhuanchen@nuaa.edu.cn

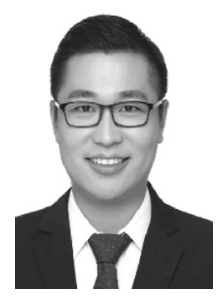

OUYANG Linhan was born in 1987. He received his Ph.D. degree from Nanjing University of Science and Technology in 2016. Now, he is an assistant professor at College of Economics and Management, Nanjing University of Aeronautics and Astronautics. His research interests are quality engineering, modeling process, and engineering optimization. E-mail: ouyang@nuaa.edu.cn 